\title{
Dynamics of Light-Field Control of Molecular Dissociation at the Few-Cycle Limit
}

\author{
X. M. Tong ${ }^{1}$ and C.D. Lin $^{2}$ \\ ${ }^{1}$ Institute of Materials Science, Graduate School of Pure and Applied Sciences, and Center for Computational Sciences, \\ University of Tsukuba, 1-1-1 Tennodai, Tsukuba, Ibaraki 305-8573, Japan \\ ${ }^{2}$ Physics Department, Kansas State University, Manhattan, Kansas 66506-2601, USA
}

(Received 9 September 2006; published 22 March 2007)

\begin{abstract}
We studied the laser-molecule interaction dynamics that leads to the asymmetric $\mathrm{D}^{+}$ion ejection in the dissociative ionization of $\mathrm{D}_{2}$ molecules observed recently in Kling et al. [Science 312, 246 (2006)]. By changing the carrier-envelope phase, we showed that the asymmetry is a consequence of manipulating the initial ionization and the rescattering of the electrons within one optical cycle of the laser. The result illustrates the feasibility of coherent control of reaction dynamics at the attosecond time scale.
\end{abstract}

Laser light with a controlled electric field $E(t)=$ $E_{0}(t) \cos (\omega t+\phi)$ has become available recently. In addition to the amplitude $E_{0}(t)$ and the frequency $\omega$, experimentally it is now possible to control the carrier-envelope phase (CEP) $\phi$. By changing the CEP, the electric field of the laser can be manipulated at the attosecond time scale. Because of the exponential dependence of tunneling ionization on the instantaneous electric field strength, control over $\phi$ has opened the opportunity to define the moment of ionization with a precision of several hundred attoseconds. The electron born within this narrow time window remains in the laser field, which, in principle, can be tailored at will. This allows for control over various rescattering processes. Examples can be found in high harmonic generation [1], in high energy above threshold ionization [2], and in nonsequential double ionization of $\operatorname{Ar}$ [3]. All of these experiments have been performed on atoms. On the other hand, steering the reaction of a molecule toward a particular configuration is the ultimate goal of coherent control in chemistry. In this Letter, we analyzed the recent experiment of Kling et al. [4] (which was featured in Ref. [5]) to show that coherent control at the attosecond time scale has been achieved by observing the asymmetric ejection of $\mathrm{D}^{+}$ ions in the dissociative ionization of $\mathrm{D}_{2}$ molecules by a few-cycle laser pulse.

Asymmetric dissociation of $\mathrm{D}_{2}{ }^{+}$or $\mathrm{HD}^{+}$by lasers is widely expected when there is an up-down asymmetry in the laser's electric field. For long pulses and a multiphoton ionization regime, an asymmetric electric field can be generated by changing the relative phase between the fundamental and the second harmonic of an optical field, and asymmetric fragmentation has been observed for such a pulse on $\mathrm{HD}^{+}$by Sheehy et al. [6], where the asymmetry had been previously predicted by Charron et al. [7]. For few-cycle pulses, asymmetric up-down electric fields can be varied by changing the carrier-envelope phase, and asymmetric dissociation of $\mathrm{HD}^{+}$or $\mathrm{D}_{2}{ }^{+}$by few-cycle pulses has been studied theoretically [8-11]. These theories predict that the resulting kinetic energies of the ions are about $2 \mathrm{eV}$ or less. In the work of Kling et al. [4], the asymmetries were observed for ions in the energy range of 3-8 eV. These ions were attributed to resulting from the dissociative ionization of $\mathrm{D}_{2}$ molecules via the rescattering mechanism. Full ab initio calculations for this two-electron process are not available today. In this Letter, we present a quantitative analysis of their experiment based on the rescattering theory by incorporating the laser field effect on the dissociation dynamics, leading to the asymmetric ejection of the $\mathrm{D}^{+}$ions.

Consider a $5 \mathrm{fs} 800 \mathrm{~nm}$ laser pulse, with a CEP of $\pi / 2$ and of $\pi$, respectively, as depicted in Fig. 1 . When a $\mathrm{D}_{2}$ molecule is placed in such a pulse, it can be ionized when the electric fields are near the local maximum, as indicated by $\mathrm{A}, \mathrm{B}, \mathrm{C}$, and $\mathrm{D}$ in the figure. The ionization releases an electron into the laser field. Meanwhile, a vibrational wave packet is created on the $1 s \sigma_{g}$ curve of $\mathrm{D}_{2}{ }^{+}$. When the electron returns at a later time to recollide with the $\mathrm{D}_{2}{ }^{+}$ ion, it may excite the ion to the $2 p \sigma_{u}$ curve. Once on the $2 p \sigma_{u}$ curve, it may be further ionized by the laser, resulting in double ionization [12-14], or it may dissociate

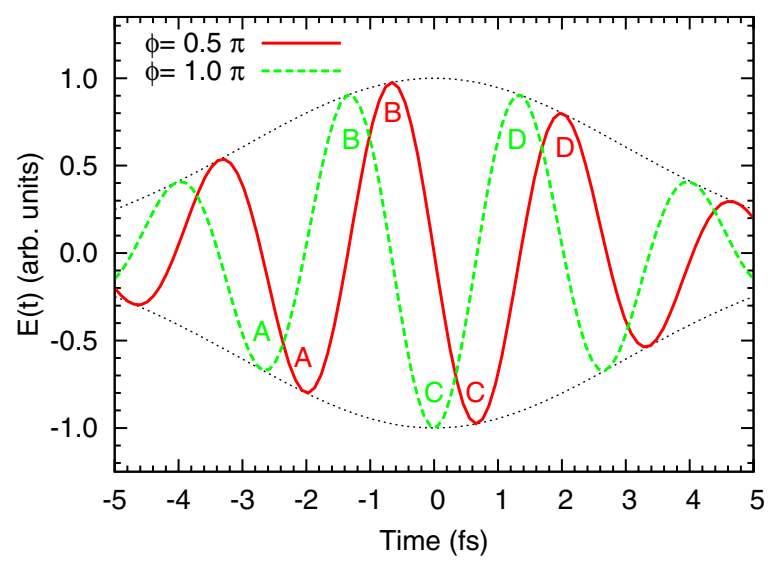

FIG. 1 (color online). Time-dependent electric field of a $5 \mathrm{fs,}$ $800 \mathrm{~nm}$ laser pulse, with CEP $\phi=\pi / 2$ (solid line) and $\phi=\pi$ (dashed lines). The letters indicate the short instants where tunneling ionization occurs. 
directly into $\mathrm{D}^{+}+\mathrm{D}$ [15]. The relative yields of dissociation vs ionization depend on the laser intensity and its duration. For the short pulses treated here, dissociation dominates, and $\mathrm{D}_{2}{ }^{+}$may dissociate directly along the $2 p \sigma_{u}$ potential curve, or the laser pulse may couple it to the $1 s \sigma_{g}$ curve and dissociate along that curve. Since these two potential curves are degenerate at large internuclear distances, the coherent sum or difference of the two scattering amplitudes gives the probability amplitude for finding the $\mathrm{D}^{+}$ions in either the up or the down direction. The relative yield, or the asymmetry, depends on the carrierenvelope phase of the pulse.

We now describe the computational steps employed in calculating the $\mathrm{D}^{+}$ejection. To begin with, at time $t_{i}$, near the laser field peaks A, B, C, and D (Fig. 1), the $\mathrm{D}_{2}$ molecule is ionized by tunneling ionization, with the rate calculated according to the molecular tunneling ionization theory [16]. The initial velocity of the electron is assumed to follow Gaussian distributions [17]. For each initial condition, the trajectory of the ionized electron in the laser

$$
i \frac{\partial}{\partial t}\left(\begin{array}{c}
C_{g}(t) \\
C_{u}(t)
\end{array}\right)=\left(\begin{array}{c}
E_{g}[R(t)]-i W_{g}[R(t)] / 2 \\
-D[R(t)] E(t)
\end{array}\right.
$$

with initial conditions $C_{u}\left(t_{r}\right)=1$ and $C_{g}\left(t_{r}\right)=0$ for $R\left(t_{r}\right)=R_{0}$. In this equation, $D(R)$ is the dipole moment of $\mathrm{D}_{2}{ }^{+}$at internuclear distance $R, E_{g, u}(R)$ are the electronic potential energies, and $W_{g, u}(R)$ are the tunneling ionization rates, of the $1 s \sigma_{g}$ and $2 p \sigma_{u}$ states, respectively, and $E(t)$ is the laser's electric field. Note that the relation between time and distance $R$ is obtained by solving the classical equation along the $2 p \sigma_{u}$ potential curve.

The probability density for finding the $\mathrm{D}^{+}$ions to be along the up or the down direction is then given by

$$
P_{d, u}\left(R_{0}, t_{r}\right)=\left|\frac{C_{g}(\infty) \pm C_{u}(\infty)}{\sqrt{2}}\right|^{2} .
$$

Note that $P_{u}\left(R_{0}, t_{r}\right)$ and $P_{d}\left(R_{0}, t_{r}\right)$ are the probabilities for finding $\mathrm{D}^{+}$ions in the up or the down direction at large distances, respectively, if the $\mathrm{D}_{2}{ }^{+}$was initially excited to the $2 p \sigma_{u}$ state at time $t_{r}$ and the internuclear distance between the two deuterons was $R_{0}$. Combining with the probability densities from tunneling ionization and the electron impact excitation probability density $P_{\text {ex }}$, we can write down the differential probability density per unit energy for finding $\mathrm{D}^{+}$in the up or the down direction by

$$
\frac{P_{d, u}(E)}{d E}=\frac{1}{C} \int \sum_{t_{r}} P_{\sigma_{u}}\left(R_{0}, t_{i}, t_{r}\right) P_{d, u}\left(R_{o}, t_{r}\right) d t_{i},
$$

with $C=d E_{u}\left(R_{0}\right) / d R_{0}$ and

$$
P_{\sigma_{u}}\left(R_{0}, t_{i}, t_{r}\right)=W_{i}\left(t_{i}\right) \chi^{2}\left(R_{0}, t_{r}-t_{i}\right) P_{\mathrm{ex}}\left(R_{0}, t_{i}, t_{r}\right) .
$$$$
\left.\begin{array}{c}
-D[R(t)] E(t) \\
E_{u}[R(t)]-i W_{u}[R(t)] / 2
\end{array}\right)\left(\begin{array}{l}
C_{g}(t) \\
C_{u}(t)
\end{array}\right),
$$

field and the potential of the $\mathrm{D}_{2}{ }^{+}$ion are calculated. By monitoring the distance of the closest approach of the electron, the return time $t_{r}$ and the kinetic energy are recorded. Using semiempirically fitted excitation cross sections, the excitation probability density by a rescattering electron at time $t_{r}$ is then calculated for a range of internuclear distances $R$ [14]. The range of $R$ to be included is given by $\left|\chi\left(R, t_{i}, t_{r}\right)\right|^{2}$, where $\chi\left(R, t_{i}, t_{r}\right)$ is the vibrational wave packet at time $t_{r}$ that was created at time $t_{i}$, assuming that the Franck-Condon principle is applicable in the ionization process. The ionization probability density for populating $\mathrm{D}_{2}{ }^{+}$ions in the excited $2 p \sigma_{u}$ state at time $t_{r}$, with internuclear distance $R_{0}$, is then calculated. If the $\mathrm{D}_{2}{ }^{+}$dissociates following the repulsive $2 p \sigma_{u}$ potential curve only, the distribution of $\mathrm{D}^{+}$would be symmetric. However, when dissociation occurs in the laser field, part of the amplitude can be transferred to the $1 s \sigma_{g}$ curve. The amplitudes for finding $\mathrm{D}_{2}{ }^{+}$in $2 p \sigma_{u}$ or $1 s \sigma_{g}$ at the end of the laser pulse are obtained by solving the coupled equations:

In this expression, we can read that $W_{i}\left(t_{i}\right)$ is the tunneling ionization probability density when the laser's electric field is $E\left(t_{i}\right) \cdot \chi^{2}\left(R_{0}, t_{r}-t_{i}\right)$ is the density of the wave packet after it has propagated over a time interval $t_{r}-t_{i}$; $P_{\text {ex }}\left(R_{0}, t_{i}, t_{r}\right)$ is the total probability of exciting $\mathrm{D}_{2}{ }^{+}$from $1 s \sigma_{g}$ to $2 p \sigma_{u}$ at the internuclear distance $R_{0}$ by the electrons. This probability is obtained after integrating over all of the trajectories of the tunneled electrons. Note that, for each initial ionization, there are more than one return times and they should be summed over. The initial ionization time $t_{i}$ can occur throughout the laser pulse and thus should be integrated over as well, even though contributions are dominated by the short time intervals marked A, B, C, and $\mathrm{D}$ in Fig. 1. The prefactor on the right-hand side converts the differential probability from per unit length to per unit energy.

To illustrate the effect of $2 p \sigma_{u}-1 s \sigma_{g}$ coupling by the laser which is responsible for generating the up-down asymmetry of $\mathrm{D}^{+}$ions, we consider a 5 fs laser pulse with peak intensity of $1.2 \times 10^{14} \mathrm{~W} / \mathrm{cm}^{2}$ and a CEP at $\pi / 2$ and $\pi$. In Fig. 2, we show the asymmetry, defined by

$$
A\left(R_{0}, t_{r}\right)=\frac{P_{u}\left(R_{0}, t_{r}\right)-P_{d}\left(R_{0}, t_{r}\right)}{P_{u}\left(R_{0}, t_{r}\right)+P_{d}\left(R_{0}, t_{r}\right)},
$$

as a function of $\left(R_{0}, t_{r}\right)$. That is, the asymmetry depends on the rescattering time $t_{r}$ and the internuclear distance $R_{0}$.

We next explore the origin of the asymmetry of the calculated $\mathrm{D}^{+}$ion yield. In Fig. 3(b), for $\phi=\pi$ we show how the up and down $\mathrm{D}^{+}$ions depend on the time of the first ionization. Major contribution to the $\mathrm{D}^{+}$ions 


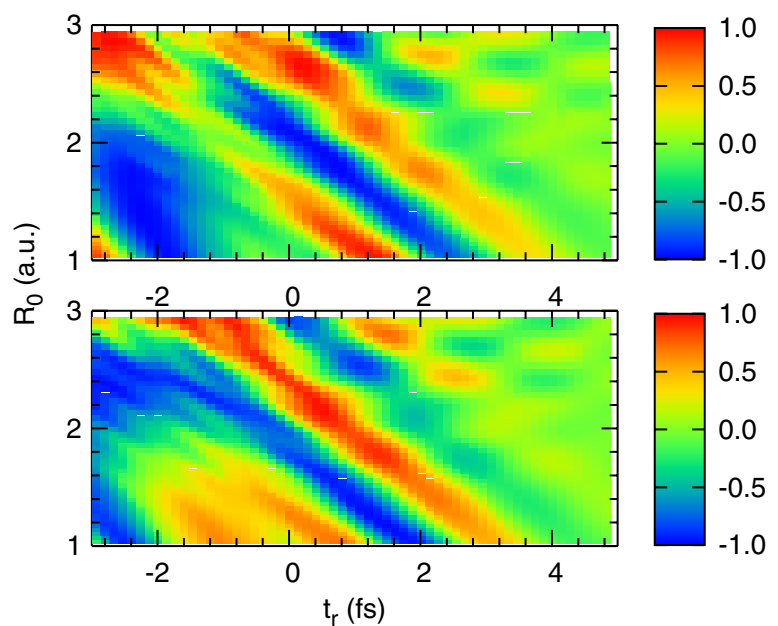

FIG. 2 (color online). The asymmetric dissociation of $\mathrm{D}_{2}{ }^{+}$. The dissociation occurs in the laser field and depends on the time $t_{r}$ (horizontal axis) when rescattering occurs and the internuclear distance $R_{0}$ (see text). Upper panel, $\phi=\pi / 2$; lower panel, $\phi=$ $\pi$.

comes from initial ionization when the laser field is near peak $\mathrm{C}$ in Fig. 1 at $t_{i}=0$. The peak kinetic energy of $6 \mathrm{eV}$ in the up direction corresponds to a return time at $t_{r}=2 \mathrm{fs}$ or at the first return. Additional contributions to the ion yield come from ionization occurring near peak B. For this group, the peak yield is also near $6 \mathrm{eV}$, again due to rescattering after the first return, but the $\mathrm{D}^{+}$ions are in the down direction. The asymmetry is due to the larger ionization rate at $\mathrm{C}$ than at $\mathrm{B}$. Note that we found little contributions from ionization near peak A, presumably due to the low tunneling ionization rate, and little from ioniza-

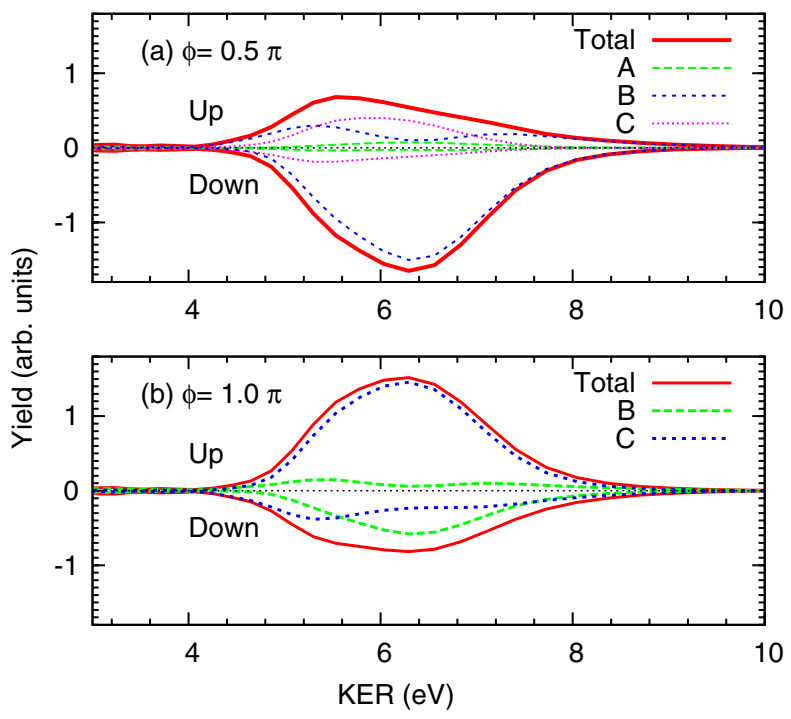

FIG. 3 (color online). Dependence of up-down ejection of $\mathrm{D}^{+}$ ions on the time of the initial ionization of $\mathrm{D}_{2}$. For the letters, refer to Fig. 1. The horizontal scale is the kinetic energy of $\mathrm{D}^{+}$ ions after dissociation. tion near peak $\mathrm{D}$, presumably due to the small kinetic energy gained in the field.

We further analyze the $\mathrm{D}^{+}$ion yield for $\phi=\pi / 2$, shown in Fig. 3(a). For this case, the electric fields at B and $\mathrm{C}$ have the same strength and, thus, the same initial ionization rates. However, electrons generated at B see a stronger electric field during the rescattering, thus gaining more kinetic energy and higher excitation probabilities. They lead to $\mathrm{D}^{+}$ions in the down direction. For electrons ionized at $\mathrm{C}$, they lead to $\mathrm{D}^{+}$ions in the up direction. We see small contributions from ionization at $\mathrm{A}$, but one can conclude that for $\phi=\pi / 2$ the asymmetry in $\mathrm{D}^{+}$ejection is due to the different kinetic energy gained during the rescattering. On the other hand, the asymmetry at $\phi=\pi$ is mostly due to the different tunneling ionization rates.

From Figs. 3(a) and 3(b), we conclude that for $\mathrm{D}_{2}$ ionized near $\mathrm{B}(\mathrm{C})$, the $\mathrm{D}^{+}$is ejected in the down (up) direction. Note that $\mathrm{D}^{+}$ions are generated only when $\mathrm{D}_{2}$ is first ionized within one optical cycle comprising peaks B and $\mathrm{C}$. By changing the CEP, we change the electric field of this cycle. This, in turn, changes the relative up-down production of $\mathrm{D}^{+}$, illustrating coherent control of chemical reactions at the single optical cycle level.

The above analysis shows that the asymmetric $\mathrm{D}^{+}$ion production depends critically on the CEP of the laser pulse. We comment that the total $\mathrm{D}^{+}$ion yield is rather insensitive to the carrier-envelope phase, even in their kinetic energy release spectra; see Fig. 4(a). The up-down asymmetry is a consequence of the coherence due to the additional coupling between the two electronic states by the laser field. In Fig. 4(b), we show the asymmetry of finding $\mathrm{D}^{+}$ions vs the CEP, by integrating over the kinetic energy release of the ions. An asymmetry of about $40 \%$ has been found. Similarly to Ref. [4], we also showed the asymmetry of the up and down $\mathrm{D}^{+}$ions on the plane of CEP and the kinetic energy of the $\mathrm{D}^{+}$ions; see Fig. 4(c).

The results presented so far are for $\mathrm{D}_{2}$ molecules aligned parallel to the laser polarization direction. We also studied the dependence of asymmetry on the alignment angle of the molecular axis with respect to the laser polarization direction. Our results show that the integrated asymmetry does not change much from $0^{\circ}$ to about $60^{\circ}$. At larger angles, the asymmetry drops quickly, and at $90^{\circ}$ the asymmetry vanishes since the laser does not couple the $1 s \sigma_{g}$ and $2 p \sigma_{u}$ states when the molecular axis is perpendicular to the polarization.

How do the present results compare to the experimental data of Kling et al. [4]? First, in Fig. 4(a), the high-energy tail of $\mathrm{D}^{+}$of about $8 \mathrm{eV}$ agrees well with the experiment, but the low-energy tail of $4 \mathrm{eV}$ is higher than the $2-3 \mathrm{eV}$ in the experiment. We believe that these low-energy ions are not due to rescattering but are mostly due to the asymmetric dissociation of $\mathrm{D}_{2}^{+}$treated previously [7-10]. Second, in Fig. 4(b), the calculation shows an asymmetry modulation depth of $80 \%$, while the experiment reported a modu- 

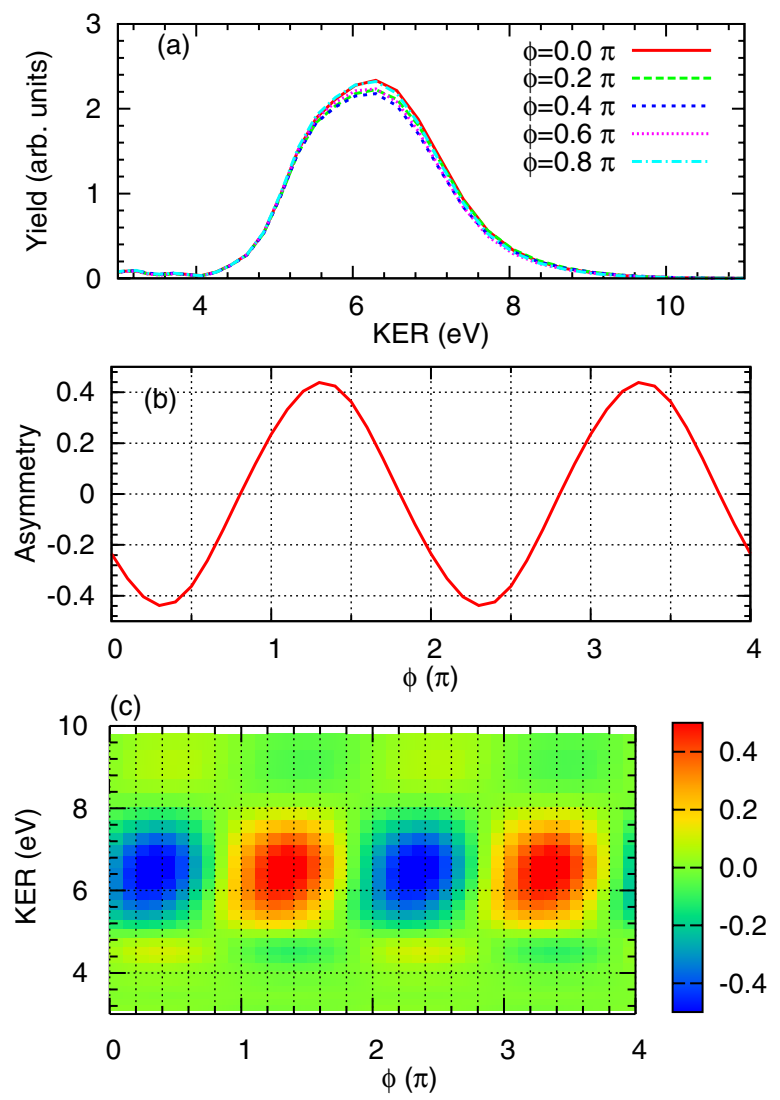

FIG. 4 (color online). (a) Total dissociative ionization spectra vs the kinetic energy release of $\mathrm{D}^{+}$ions for different carrierenvelope phases. (b) The dependence of up-down asymmetry of $\mathrm{D}^{+}$ions on the carrier-envelope phase. (c) The dependence of up-down asymmetry on the $\mathrm{D}^{+}$ion energy and the carrierenvelope phase.

lation depth of $50 \%$. The calculation shown was for a $5 \mathrm{fs}$ (FWHM) Gaussian pulse. For a 6 fs pulse, our calculation gave a modulation depth of $60 \%$. Our extended calculations also showed that the up-down asymmetry depends on the pulse shape. The near-degeneracy between $1 s \sigma_{g}$ and $2 p \sigma_{u}$ at large $R$ implies that even a small electric field would couple the two channels, and, thus, the tail of the laser pulse would affect the asymmetry. In view of these uncertainties, we consider the agreement with the experimental data reasonable. Third, our calculation shows how the asymmetry depends on the carrier-envelope phase, while in the experiment the actual phase was not measured and the asymmetry was shown with respect to the relative phase only.

In conclusion, our analysis establishes firmly that the asymmetric $\mathrm{D}^{+}$ion ejection in the dissociative ionization of $\mathrm{D}_{2}$ molecules observed by Kling et al. is a consequence of singe-cycle dynamics even though a 5 fs pulse has been used. The asymmetry depends strongly on the carrierenvelope phase. Because of the exponential dependence of tunneling ionization, initial ionization occurs in the attoseconds scale at a specific half-cycle near where the laser field is at the peak. In the future, it is desirable to measure the energy of the electrons in coincidence with the $\mathrm{D}^{+}$ions. Such a measurement would identify electrons ejected within half an optical cycle directly and their role in the rescattering process. From this analysis, we showed that, by controlling the carrier-envelope phase, the ionization of $\mathrm{D}_{2}$ and the subsequent dissociation of $\mathrm{D}_{2}{ }^{+}$can be fully controlled at the single-cycle limit. This exemplifies coherent control at the shortest time scale available today.

C.D.L. is supported in part by Chemical Sciences, Geosciences and Biosciences Division, Office of Basic Energy Sciences, Office of Science, U.S. Department of Energy.

[1] A. Baltska et al., Nature (London) 421, 611 (2003).

[2] G. Paulus et al., Phys. Rev. Lett. 91, 253004 (2003).

[3] X. Liu et al., Phys. Rev. Lett. 93, 263001 (2004).

[4] M.F. Kling, C. Siedschlag, A. J. Verhoef, J. I. Khan, M. Schultze, T. Uphues, Y. Ni, M. Uiberacker, M. Drescher, F. Krausz, and M. J. J. Vrakking, Science 312, 246 (2006).

[5] C. Day, Phys. Today 59, 13 (2006).

[6] B. Sheehy, B. Walker, and L. F. DiMauro, Phys. Rev. Lett. 74, 4799 (1995).

[7] E. Charron, A. Giusti-Suzor, and F. H. Mies, Phys. Rev. Lett. 71, 692 (1993).

[8] V. Roudnev, B. D. Esry, and I. Ben-Itzhak, Phys. Rev. Lett. 93, 163601 (2004).

[9] A. D. Bandrauk, S. Chelkowski, and H. S. Nguyen, Int. J. Quantum Chem. 100, 834 (2004).

[10] I. Kawata, H. Kono, and Y. Fujimura, J. Chem. Phys. 110, 11152 (1999).

[11] G. L. Yudin, S. Chelkowski, J. Itatani, A. D. Bandrauk, and P. B. Corkum, Phys. Rev. A 72, 051401(R) (2005).

[12] A. S. Alnaser, T. Osipov, E. P. Benis, A. Wech, B. Shan, C. L. Cocke, X. M. Tong, and C. D. Lin, Phys. Rev. Lett. 91, 163002 (2003).

[13] X. M. Tong, Z. X. Zhao, and C. D. Lin, Phys. Rev. Lett. 91, 233203 (2003).

[14] X. M. Tong, Z. X. Zhao, and C. D. Lin, Phys. Rev. A 68, $043412(2003)$.

[15] H. Niikura, F. Legare, R. Hasbani, A. D. Bandrauk, M. Y. Ivanov, D. M. Villeneuve, and P. B. Corkum, Nature (London) 417, 917 (2002).

[16] X. M. Tong, Z. X. Zhao, and C. D. Lin, Phys. Rev. A 66, 033402 (2002).

[17] G. L. Yudin and M. Y. Ivanov, Phys. Rev. A 63, 033404 (2001). 